\title{
The efficiency of high powered diode laser in root canal disinfection - a
}

case report

\section{Elvira Gyulbenkiyan}

Department of Conservative Dentistry, Faculty of Dental Medicine, Medical University - Sofia, Bulgaria;

\begin{abstract}
The aim of this case report is to describe a mandibular second molar with periapical lesion and rare anatomy of the endodontic system which was treated with additional method of root canal disinfection. Intra oral radiograph revealed a tooth with not exacting endodontic treatment and with relatively large apical radiolucency. The additional disinfection performed after the root canal preparation was finished with diode laser for $20 \mathrm{sec}$. The 18-month follow-up revealed complete resolution of the periapical radiolucency.
\end{abstract}

Keywords: apical periodontitis, retreatment, rare endodontic anatomy, high powered diode laser.

\section{Introduction}

Due to pulp necrosis, the root canal system becomes increasingly susceptible to colonization by the microorganisms that inhabit the oral cavity and interconnected systems. The close physiopathological relationship between the pulp and the periapical region predisposes bacteria, fungi, and cell components to inflamed the periapical tissues and progressively to affect them through the resorption process (1).

This case report presents an endodontically treated mandibular second molar tooth with apical periodontitis. The tooth is with a rare root canal system, a single root with a single canal. In the literature is very little 
mentioned about single canal with a round or oval orifice in a second mandibular molars $(2,3)$. The literature has indicated that the success of retreatment of teeth with apical periodontitis is lower, with an overall success rate of $66 \%$ (4). The success of endodontic treatment depends on cleaning, shaping and sealing the root canal system (5) and on good knowledge of the anatomy and the variations in the human dentition (6).

Unsuccessful endodontic treatment is one of the reason for surgical procedures - incision, periapical surgery, amputation, hemisection or eventual tooth extraction. Therefore, the effective disinfection and decontamination play a key role in the root canal treatment (7). In the last years, diode lasers are used successfully as additional method of root canal disinfection especially in the case of apical periodontitis (8). Laser light can penetrate areas of canals where irrigating and disinfecting solutions cannot reach, like secondary canals and deep dentinal tubules and also can eliminate microorganisms. Diode laser at wave length of $940 \mathrm{~nm}$ could reduce intracanal bacterial count and penetrate in the depth of 500 microns in dentin $(9,10)$.

The purpose of this case report is to report a mandibular second molar with periapical lesion and rare anatomy of the endodontic system which was treated with additional method of root canal disinfection.

\section{Case Report}

A 32 year old female was referred for endodontic treatment with pain in relation to her mandibular left second molar. The tooth on examination revealed a large restoration. The patient had pain on percussion. Intra oral radiograph revealed a tooth with not exacting endodontic treatment and with relatively large apical radiolucency. The root canal morphology confirmed the presence of a single root with a linear canal, constricting towards the apex (figure 1).

Diagnosis: previously treated, symptomatic apical periodontitis.

Treatment is nonsurgical endodontic retreatment followed by permanent restoration of the access cavity.

Under rubber dam isolation an access cavity was prepared. Only a single round orifice was located in the middle portion of the floor of the pulp chamber. The retreatment was done using crown-down technique $(\mathrm{H}-$ files, Dentsply, Maillefer, Switzerland). Cleaning and shaping was realized by rotary instrumentation ProTaper Universal \#40, (Dentsply, Maillefer, Switzerland) (figure 2). A thorough irrigation with sodium hypochlorite $2,5 \%$ and saline was done after each instrument throughout the procedure. The additional disinfection performed after the root canal preparation was finished with diode laser ( $940 \mathrm{~nm}, 0.1 \mathrm{~W}$, tip E2; Epic-Biolase, Irvine, CA, USA) for $20 \mathrm{sec}$. The canal was obturated using thermo-plasticized injectable gutta-percha (Bee Fill, VDW, GmbH, Germany). 


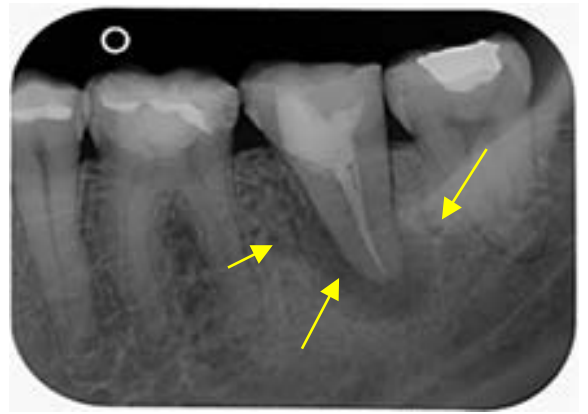

Fig. 1. Radiograph of tooth 37 before treatment (PAI 5)

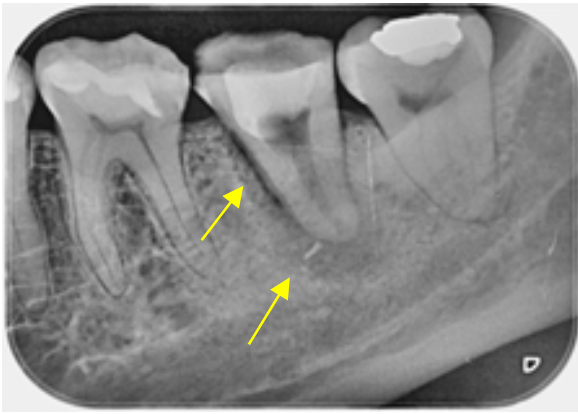

Fig. 2. Radiograph of tooth 37 during treatment (PAI 5)

An 18-month follow-up was done using radiographic examination (figures $3-6$ ), as the analysis of the periapical reduction was evaluated with Periapical Index (PAI) by Ǿstravik (11) (table 1). For a year and a half the periapical lesion reduces from PAI 5 to PAI 2 as the tooth remains asymptomatic. Further a prosthetic treatment is needed to protect the root from fracture.

Table 1. Periapical Index (Ǿstravik et al.)

PAI Score Description of Radiographic findings

1

2

3

4

5
Normal Periapical Structures

Small changes in Bone Structures

Change in bone Structures with Mineral Loss

Periodontitis with well-defined radiolucent area

Severe periodontitis with exacerbating features 


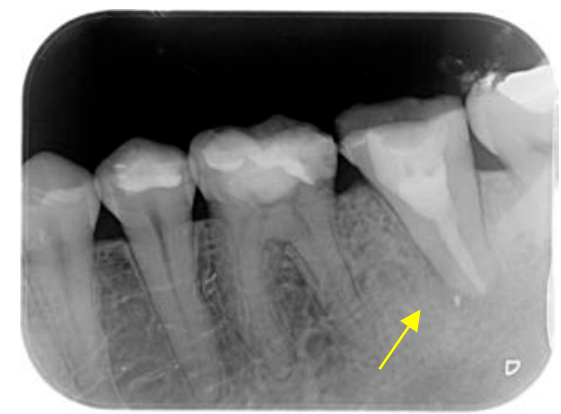

Fig. 3. 3th-month follow-up of tooth 37 (PAI 3)

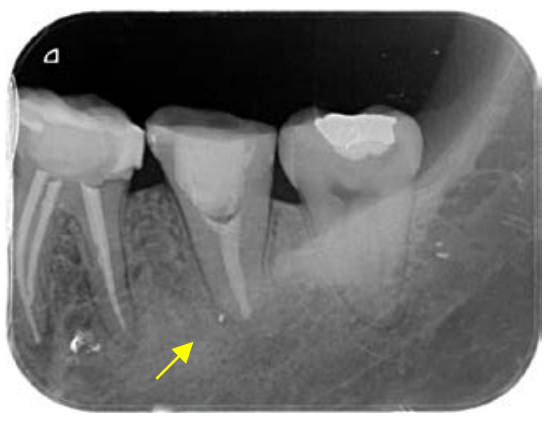

Fig. 5. 12th-month follow-up of tooth 37 (PAI 2)

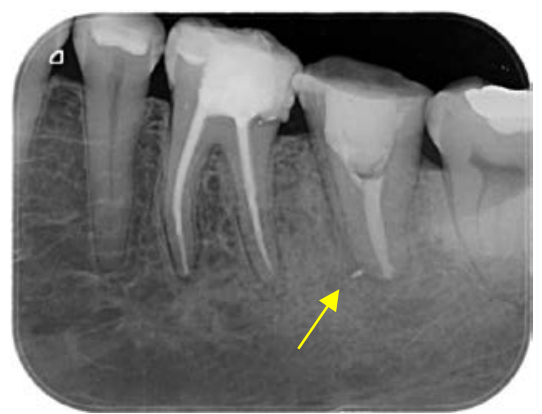

Fig. 4. 6th-month follow-up of tooth 37 (PAI 2)

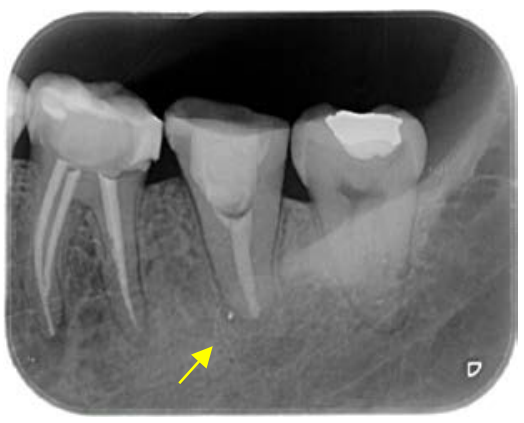

Fig. 6. 18th-month followup of tooth 37 (PAI 2)

\section{Discussion}

We found the presence of a single root with a single, non-conical canal in the mandibular second molar in our case which is quite rare (12). In the literature is reported more often the presence of C-shaped canals among the mandibular second molars and these configurations are mostly seen among single rooted mandibular molars (13). A thorough understanding of the complexity of the root canal system is essential for the clinical and radiographic outcome. The collection of retrospective and prospective literature on retreatments has revealed variable success percentages ranging between $40 \%$ and $85 \%$ (14). Studies performed in relation to the antimicrobial effect of diode laser with different parameters showed that this laser can perform high effectiveness in reduction of intra canal bacteria and penetrates to a depth of 500 microns in dentin $(9,10)$. The thorough irrigation during endodontic treatment is crucial for the final success. In our clinical case we used the ability of the diode laser in particular the laser beam deep to penetrate in dentinal tubules, where the most commonly used irrigating solutions, activated or nonactivated with different methods cannot reach (15).

\section{Conclusion}

The effective disinfection and decontamination have a central role in endodontic treatment to stop the inflammatory processes and to allow the regeneration of periodontium. 


\section{References}

1. Soares J.A., M Leonardo, L Silva et al. Histomicrobiologic aspects of theroot canal system and periapical lesions in dogs' teethafter rotary instrumentation and intracanal dressingwith $\mathrm{Ca}(\mathrm{OH}) 2$ pastes. J Appl Oral Sci, 2006;14, 355-364.

2. Fava L.R., I. Weinfeld, F.P. Fabri et al. Four second molars with single roots and single canals in the same patient. Int Endod J 2000;33:138-42.

3. Shetty N., V. Singh, S. Rijal. Single rooted mandibular second molars with single canal: Rare occurance. Endodontology 2000;2:55-9.

4. Fabio G., M. Gorni, M. Gagliani. The Outcome of Endodontic Retreatment: A 2-yr Follow-up., Jour of Endod., 2004, Jan., 30 (1).

5. Haapasalo M., D. Ørstavik. In vitro infection and disinfection of dentinal tubules., J Dent Res, 1987; 66:1375-9.

6. Fan W., F. Bing, J. Gutmann et al. Identification of a C-shaped canal system in mandibular second molars-Part III:Anatomic features revealed by digital substration radiography., 2008, J Endod; 34:1187-1190.

7. Gusiyska A., E. Gyulbenkiyan, R. Vassileva et al. Effective root canal irrigation - a key factor of endodontic treatment - review of the literature. International Journal of Recent Scientific Research 2016; 7(4):9962-9970.

8. Gueorgieva Tz., M. Dencheva, J. Kamenoff et al. A comparative study of microleakage of obturated root canals after disinfection with Nd:Yag laser, PDT and NAOCL and EDTA. J of IMAB; 2013; 19: 415-418.

9. Kreisler M., W. Kohnen, M. Beck et al. Efficacy of $\mathrm{NaOCl} / \mathrm{H} 2 \mathrm{O} 2$ irrigation and GaAlAs laser in decontamination of root canals in vitro. Lasers Surg Med. 2003;32:189-96.

10. Moritz A., N. Gutknecht, K. Goharkhay et al. In vitro irradiation of infected root canals with a diode laser: results of microbiologic, infrared spectrometric and stain penetration examinations. Quintessence Int. 1997;28:205-9.

11. Ørstavik D., K. Kerekes, H. M. Eriksen. The periapical index: a scoring system for radiographic assessment of apical periodontitis. Endod Dent Traumatol. 1986; Feb;2(1):20-34.

12. Ng Y.L.,T Aung, A Alavi et al. Root and canal morphology of Burmese maxillary, mandibular molars. Int Endod J. 2001 Dec; 34(8): 620-30.

13. Rahimi S, S. Shahi, M. Lotfi et al. Root canal configuration and the prevalence of C-shaped canals in mandibular second molars in an Iranian population. J Oral Sci 2008;50:9-13.

14. Torabinejad M, R. Corr, R. Handysides et al., Review of Nonsurgical Retreatment and Endodontic Surgery. J Endod. 2009 Jul;35(7):930-937

15. Ghorbanzadeh A, M. Aminsobhani, K. Sohrabi al. Penetration Depth of Sodium Hypochlorite in Dentinal Tubules after Conventional Irrigation, Passive Ultrasonic Agitation and Nd:YAG Laser Activated Irrigation. J Lasers Med Sci, 2016, 7(2).

\section{Corresponding author:}

Elvira Gyulbenkiyan,

Department of Conservative Dentistry and Endodontics,

Faculty of Dental Medicine,

Medical University-Sofia, Bulgaria,

Tel: +359898348535

email: elvira.simon@gmail.com 